\title{
Single coronary artery, and dissecting aneurysm of ascending aorta following resection of coarctation of aorta
}

\author{
J. D. A L DER SON \\ Cardio-thoracic Unit, Royal Infirmary, Sheffield
}

\begin{abstract}
A patient who developed a dissecting aneurysm of the ascending aorta 14 years after satisfactory resection of a coarctation of the aorta is reported. He was found to have an absent right coronary artery at necropsy following unsuccessful excision and repair of the aneurysm.
\end{abstract}

\section{CLINICAL HISTORY}

A 28-year-old kitchen hand was admitted in September 1969 for exploration of a suspected aortic aneurysm. He gave a history of two attacks of chest pain associated with breathlessness for which he was hospitalized in late 1968. At that time he was noted to have a systolic murmur suggestive of aortic stenosis and had signs of heart failure. He ran a low-grade pyrexia and had a persistently raised E.S.R., but blood cultures were negative.

Past medical history revealed that at the age of 14 years he had had a resection of basal segments of the left lower lobe and the lingula for bronchiectasis, and a resection of a normally sited aortic coarctation with primary anastomosis, both procedures being performed at the same operation. Following this he had maintained fairly good health.

In early 1969 he was readmitted because of persistent low-grade fever, elevated E.S.R., and the development of a new aortic diastolic murmur, and was treated for subacute bacterial endocarditis although blood cultures had again been negative.

Later in that year he was admitted for cardiac catheterization, having been relatively asymptomatic since the previous discharge. There was evidence of left ventricular hypertrophy together with an ejection type systolic murmur in the left third interspace, radiating into the neck, and an easily heard ejection click. There was a faint diminuendo diastolic murmur down the left sternal edge.

Cardiac catheterization (Seldinger technique) revealed a systolic gradient of $30-40 \mathrm{mmHg}$ at the aortic valve. Cineangiography revealed a normal left ventricle and descending aorta. Biplane angiography revealed a large aneurysmal sac of the ascending aorta, the neck of which was thought to be small.

Unfortunately the left ventricle could not be entered for technical reasons and the coronary arteries were not visualized.
On admission in September 1969 for exploration of the aortic aneurysm further history revealed that the $\theta$ patient had had occasional right-sided attacks of chest $N$ pain for several years with palpitations. He was not $\square$ breathless but suffered from an intermittently productive cough with greenish sputum, with an episode of haemoptysis two years before admission. He smoked 10 cigarettes each day.

Examination revealed a thin man with a high $\stackrel{\mathbb{D}}{\circ}$ palate. There was a left thoracotomy scar. The pulse $\overrightarrow{\overrightarrow{0}}$ rate was $80 /$ minute, regular; blood pressure $90 / 65 \frac{3}{3}$ $\mathrm{mmHg}$. Jugular venous pressure was raised. There was a visible left ventricular impulse and the apex beat was in the sixth intercostal space in the midclavicular line. There was a palpable systolic thrill $\overrightarrow{7}$ in the aortic area. All peripheral pulses were present, equal, and of good volume. Heart sounds I and II were audible with a loud grade IV systolic murmur, 윽 maximal in the aortic area and radiating into the neck.

The diagnosis of aortic stenosis and of dissecting aneurysm of the ascending aorta, probably partially $ᄋ$ obstructing the superior vena cava, was made.

A blood film revealed reduced numbers of platelets, $\frac{D}{0}$ but was otherwise normal. Serum electrolytes, plasma proteins, liver function tests, and bleeding and $\mathcal{O}$ clotting times were within normal limits. The electrocardiogram showed left ventricular hyper- $N$ trophy.

\section{OPERATIVE FINDINGS}

At operation, following a routine median sternotomy, it was apparent that there was a large aneurysm of the ascending aorta, densely adherent to the $\frac{0}{0}$ pericardium and pressing upon the superior vena $\frac{+}{\Phi}$ cava, right heart outflow tract, pulmonary artery, and the greater part of the right atrium.

It was possible after opening the pericardium to expose a small portion of the right atrium, in relation 
to the inferior vena cava, and a single cannula was inserted. Standard cannulation of the left femoral - artery was performed, and perfusion was begun using hypothermia at approximately $30^{\circ} \mathrm{C}$. The aorta was controlled immediately proximal to the innominate artery and clamped. On opening the aorta it was apparent that the aneurysm was of the dissecting type with a defect $3 \mathrm{~cm}$ above the aortic ring on the left lateral margin measuring $3 \times 2 \mathrm{~cm}$. Initial repair with a Dacron patch was unsatisfactory, with haemorrhage posteriorly, therefore the dissected portion was cut back to within $1 \mathrm{~cm}$ of the aortic ring, creating a defect of approximately $4 \mathrm{~cm}$. The left coronary artery was perfused but the right coronary artery orifice could not be found. A Dacron graft was then inserted with good haemostasis distally, but the aorta proximally was of extremely poor material, and even with the support of the aneurysmal sac around the anastomosis the haemorrhage could not be controlled. It was reluctantly accepted that adequate repair could not be obtained. It was also noted at operation that the aortic valve was bicuspid with lateral fusion of the cusps but the orifice appeared to be adequate.

\section{NECROPSY FINDINGS}

Necropsy confirmed the operation findings and absence of the right coronary artery, it being represented by a small dimple. The left coronary artery was normal but had a large circumflex branch. The aortic valve was bicuspid, with slight fusion of the cusps, but other valves were normal. There was hypertrophy of the left side of the heart, but no dilatation or evidence of infarction. The coarctation repair appeared satisfactory. Examination of the left lung showed a scarred left lower lobe dorsal segment.

Histology of the aortic aneurysm revealed almost complete loss of the elastic tissue and the wall consisted of collagen. Sections of the descending aorta at the site of the coarctation were normal, but sections of the arch showed some fragmentation of the elastic tissue and an excess of collagen.

Sections of the left lower lobe remnant showed dilated bronchi consistent with bronchiectasis.

The myocardium was normal, and there was no evidence to support a previous diagnosis of subacute bacterial endocarditis.

\section{DISCUSSION}

Previous work by Blake, Manion, Mattingly, and Baroldi (1964) and Edwards (1958) has classified anomalies of the coronary arteries into those of major and minor significance. Under this classification a single coronary artery, as in this case, is regarded as minor, as it is not life threatening. Murphy (1967) noted that approximately one-third of reported cases of single coronary artery had associated serious congenital abnormalities and proposed that these accounted for the high incidence, and approximately $50 \%$ mortality rate, of cases under 20 years of age. The aetiology of single coronary artery is not, as yet, understood (Roberts and Loube, 1947).

The importance of recognizing anomalies of coronary arteries pre-operatively was emphasized in this case in that time was wasted in looking for the right coronary artery in order to establish coronary perfusion. Several instances have been recorded of accidental single coronary artery ligation during surgery (Murphy, 1967). In our case technical angiography difficulties prevented the Kifa catheter from entering the left ventricle and hence the coronary arteries could not be outlined. The electrocardiogram provided no clue, but nine previously reported cases have shown evidence of myocardial ischaemia, or even infarction (Laurie and Woods, 1964).

The sudden development of the dissecting aneurysm of the ascending aorta 14 years after resection of the coarctation has not been explained. A survey of postoperative aneurysms following surgery for coarctation of the aorta conducted by Davis, Fell, and Taylor (1965) revealed that they are rare and usually occur within one year of operation, and commonly occur at the site of the anastomosis. In all cases there was a loss of continuity of the medial elastic fibres, as was found in this case. Cases previously reported suggest that the damage usually occurs at the site of coarctation resection, presumably due to blood entering the bare medial layer produced following operation and causing the deposition of inelastic collagen, which, being unable to resist pulsatile stress, progressively distends. In our case the coarctation site was reported to be satisfactory at necropsy. However, Steinberg, Stein, and Goldberg (1965) report a case in which a mild aortic sinus aneurysm noted before operation for coarctation of the aorta had enlarged to such an extent 10 years later that moderate to severe aortic incompetence was produced.

Wagenvoort, Neufeld, and Edwards (1962) have previously pointed out that the medial degeneration of Marfan's syndrome is indistinguishable from idiopathic medial degeneration of the aorta, and Steinberg et al. (1965) have postulated that, as aortic sinus aneurysm is common in Marfan's syndrome, this may be the cause of aneurysm in patients with coarctation. Further evidence to support this in our patient is that he was noted to have a high palate preoperatively.

Bicuspid valves are a common feature of coarctation, and Hamilton and Abbott (1928) record an incidence of $25 \%$. This is known to predispose 
to stenosis (Bentivoglio, Sagarminaga, Uricchio, and Goldberg, 1960).

The author is indebted to Mr. D. G. Taylor, consultant thoracic surgeon to the Sheffield Cardiothoracic Unit, Royal Infirmary, Sheffield, for his help and encouragement and useful criticism in the preparation of this report; also to Sir Thomas Holmes Sellors for permission to use his case notes relating to the earlier operation.

Acknowledgements are also due to Dr. R. G. Grainger, consultant radiologist to the Sheffield Cardio-thoracic Unit at the Northern General Hospital, Sheffield, for interpreting the radiographs.

\section{REFERENCES}

Bentivoglio, L. G., Sagarminaga, J., Uricchio, J., and Goldberg, H. (1960). Congenital bicuspid aortic valves: a clinical and haemodynamic study. Brit. Heart J., 22, 321.

Blake, H. A., Manion, W. C., Mattingly, T. W., and Baroldi, G. (1964). Coronary artery anomalies, Circulation, 30, 927.
Davis, C. B., Fell, E. H., and Taylor, C. B. (1965). Post $-\frac{S}{-}$ operative aneurysm following surgery for coarctation of aorta. Surg. Gynec. Obstet., 121, 1043.

Edwards, J. E. (1958). Anomalous coronary arteries with $\frac{\bar{\omega}}{\partial}$ special reference to arteriovenous-like communications. $\mathbb{D}$ Circulation, 17, 1001.

Hamilton, W. F., and Abbott, M. E. (1928). Coarctation of the aorta of the adult type. Amer. Heart J., 3, 381.

Laurie, W., and Woods, J. D. (1964). Single coronary artery. A report of two cases. Amer. Heart J., 67, 95.

Murphy, M. L. (1967). Single coronary artery. Amer. Heart J. 74, 557.

Roberts, J. T., and Loube, S. D. (1947). Congenital single N coronary artery in man. Amer. Heart J., 34, 188.

Steinberg, I., Stein, H. L., and Goldberg, H. P. (1965).e Aneurysms complicating the postoperative course of coarctation of the aorta. Report of 3 cases. Amer. J. Roentgenol., 93, 331.

Wagenvoort, C. A., Neufeld, H. N., and Edwards, J. EC (1962). Cardiovascular system in Marfan's syndrome? and in idiopathic dilatation of the ascending aorta Amer. J. Cardiol., 9, 496. 